PROCEEDINGS OF THE

AMERICAN MATHEMATICAL SOCIETY

Volume 132, Number 7, Pages 1925-1936

S 0002-9939(04)07415-5

Article electronically published on January 26, 2004

\title{
EULER NUMBER OF THE MODULI SPACE OF SHEAVES ON A RATIONAL NODAL CURVE
}

\author{
BAOSEN WU \\ (Communicated by Michael Stillman)
}

\begin{abstract}
In this paper, we use finite group actions to compute the Euler number of the moduli space of rank 2 stable sheaves on a rational nodal curve.
\end{abstract}

\section{INTRODUCTION}

Although the Betti numbers of the moduli space of vector bundles on a smooth curve have been obtained by various methods, in general, they are not known for a singular curve. Let $C$ be a rational curve with nodes as singularities. There is a moduli space $\mathbf{M}$ of rank 2 stable sheaves $\mathcal{E}$ on $C$ such that $\chi(\mathcal{E})=1$. In this paper, we shall compute the Euler number of this moduli space. The result is

Main Theorem. The Euler number $e(\mathbf{M})$ is equal to $n$, the number of nodes on $C$.

As is well known, there is a canonical action of the Jacobian $J C$ on the moduli space $\mathbf{M}$, defined by tensorization. For simplicity, we find a series of finite cyclic subgroups $G$ of $J C$, and study the finite group actions. The topological nature of $\mathbf{M}$ can be reflected by these group actions.

\section{Preliminaries}

In this section, we review some basic definitions and known results.

Let $\mathcal{E}$ be a coherent sheaf on a Noetherian scheme $X$. Recall that the support of $\mathcal{E}$ is the closed set $\operatorname{Supp}(\mathcal{E})=\left\{x \in X \mid \mathcal{E}_{x} \neq 0\right\}$, and its dimension is called the dimension of the sheaf $\mathcal{E}$, denoted by $\operatorname{dim} \mathcal{E}$.

Definition 1.1. Let $X$ be a Noetherian scheme. A coherent sheaf $\mathcal{E}$ on $X$ is pure of dimension $d$ if every nonzero coherent subsheaf of $\mathcal{E}$ has dimension $d$.

Recall that a coherent sheaf $\mathcal{E}$ on an integral scheme $X$ is torsion free if for each $x \in X, m \in \mathcal{E}_{x}$, and nonzero element $s \in \mathcal{O}_{x}$ the equality $s m=0$ implies that $m=0$. Thus when $X$ is integral and $\operatorname{dim}(X)=d$, a sheaf is pure of dimension $d$ if and only if it is torsion free.

Let $\mathcal{E}$ be a torsion free sheaf on an integral scheme of dimension $d$. The maximal subsheaf of dimension $\leq d-1$ of $\mathcal{E}$, denoted by $\mathcal{E}_{\text {tor }}$, is called the torsion part of $\mathcal{E}$, and the quotient sheaf $\mathcal{E} / \mathcal{E}_{\text {tor }}$ is torsion free.

Received by the editors November 1, 2001 and, in revised form, April 17, 2003.

2000 Mathematics Subject Classification. Primary 14D20, 14 F05.

Key words and phrases. Moduli space, Euler number, group action.

(C)2004 American Mathematical Society 
Definition 1.2. We fix an ample line bundle $\mathcal{O}(1)$ on $X$. Then the Hilbert polynomial $P_{\mathcal{E}}(n)$ of $\mathcal{E}$ is given by

$$
n \rightarrow \chi(\mathcal{E} \otimes \mathcal{O}(n)) .
$$

The coefficient of the leading term of $P_{\mathcal{E}}(n)$ is $r n^{d} / d$ ! with $r$ an integer, the rank of $\mathcal{E}$. The reduced Hilbert polynomial $p_{\mathcal{E}}(n)$ is defined to be

$$
p_{\mathcal{E}}(n)=\frac{P_{\mathcal{E}}(n)}{r} \text {. }
$$

Now we come to the definition of stability of a pure sheaf.

Definition 1.3. A pure sheaf $\mathcal{E}$ is semistable if for any proper subsheaf $\mathcal{F}$ of $\mathcal{E}$ one has $p_{\mathcal{F}}(n) \leq p_{\mathcal{E}}(n)$ for sufficiently large $n$. $\mathcal{E}$ is called stable if " $\leq$ " is replaced by " $<$ ". If $\mathcal{E}$ is semistable but not stable, then it is called strictly semistable.

If $\mathcal{E}$ is strictly semistable, we consider those subsheaves $\mathcal{F} \subset \mathcal{E}$ for which equality holds above. Let $0 \subset \mathcal{F}_{1} \subset \cdots \subset \mathcal{F}_{n}=\mathcal{E}$ be a maximal chain of such sheaves. Define $\operatorname{gr} \mathcal{E}$ to be

$$
\bigoplus_{i=1}^{n} \mathcal{F}_{i} / \mathcal{F}_{i-1}
$$

This is independent of the choice of the maximal chain. We say $\mathcal{E}$ and $\mathcal{F}$ are $S$-equivalent if $g r \mathcal{E} \cong g r \mathcal{F}$.

The following theorem ensures the existence of moduli spaces (see [4] and [6]).

Theorem 1.4. Let $X$ be a projective scheme over $\mathbb{C}$, and let $\mathcal{O}_{X}(1)$ be an ample line bundle on $X$. Then there exists a (coarse) moduli space $\mathbf{M}(P)$ of semistable sheaves with Hilbert polynomial $P$. Closed points in $\mathbf{M}(P)$ correspond one-to-one to $S$-equivalence classes of semistable sheaves. Moreover, $\mathbf{M}(P)$ is projective.

Let $C$ be a rational curve with only nodes $x_{1}, x_{2}, \cdots, x_{n}$ as singularities. The following two lemmas about the torsion free sheaves on $C$ are well known (see [5]).

Lemma 1.5. Let $\mathcal{E}$ be a rank 2 torsion free sheaf on $C$, and $x$ a node on $C$. Then there exists an isomorphism of $\mathcal{O}_{x}$-modules $\psi: \mathcal{E}_{x} \cong a \mathcal{O}_{x} \oplus(2-a) m_{x}$, where $0 \leq a \leq 2$ and $m_{x}$ is the maximal ideal of $\mathcal{O}_{x}$. Therefore we have a morphism $g: \mathcal{E} \rightarrow a \mathbb{C}_{x}$, which depends on the isomorphism $\psi$, but the kernel $\mathcal{E}^{\prime}$ of $g$ is independent of this isomorphism.

Lemma 1.6. Suppose $\mathcal{F}$ is a rank 2 torsion free sheaf on $C$, and $\hat{\pi}: \hat{C} \rightarrow C$ is a partial normalization of $C$ at the nodes $x_{1}, x_{2}, \cdots x_{r}, r \leq n$. Then there exists a sheaf $\mathcal{E}$ on $\hat{C}$ such that

$$
\mathcal{F} \cong \hat{\pi}_{*} \mathcal{E}
$$

if and only if

$$
\mathcal{F}_{x_{i}} \cong 2 \cdot m_{x_{i}}
$$

for $i=1, \cdots, r$

Now let $\mathbf{M}$ be the moduli space of rank 2 stable sheaves $\mathcal{E}$ on $C$ such that $\chi(\mathcal{E})=1$. For $0 \leq a_{i} \leq 2$, let

$$
\mathbf{M}\left(a_{1}, \cdots, a_{n}\right)=\left\{\mathcal{E} \in \mathbf{M} \mid \mathcal{E}_{x_{i}} \cong a_{i} \mathcal{O}_{x_{i}} \oplus\left(2-a_{i}\right) m_{x_{i}}\right\} ;
$$

then $\mathbf{M}=\bigsqcup \mathbf{M}\left(a_{1}, \cdots, a_{n}\right)$. This gives rise to a stratification of $\mathbf{M}$.

Since our aim is to compute the Euler number $e(\mathbf{M})$, we need the following lemma for our calculations. 
Lemma 1.7. Let $X$ be an algebraic variety. Suppose that for an arbitrary large number $n$, we have a finite abelian group $G$ of order $n$, and a $G$-action on $X$ that is free of fixed points. Then the Euler number $e(X)$ is zero.

\section{The GenERALIZED JACOBIAN}

Before we study the moduli space, we first consider the Jacobian of the curve.

Let $C$ be a rational nodal curve with one node $p$, and $\pi: \tilde{C} \rightarrow C$ the normalization of $C$. Let $\tilde{\mathcal{O}}_{p}$ be the integral closure of $\mathcal{O}_{p}$. We use $*$ to denote the group of units in a ring. From the exact sequence

$$
0 \rightarrow \tilde{\mathcal{O}}_{p}^{*} / \mathcal{O}_{p}^{*} \rightarrow J C \rightarrow J \tilde{C} \rightarrow 0
$$

and $\tilde{\mathcal{O}}_{p}^{*} / \mathcal{O}_{p}^{*} \cong \mathbb{C}^{*}, \tilde{C} \cong \mathbb{P}^{1}$, we have $J C \cong \mathbb{C}^{*}$.

Now we shall derive the same result from another point of view, which can be generalized.

Note that $\pi_{*} \mathcal{O}_{\tilde{C}}$ is a torsion free but not locally free sheaf on $C, \pi_{*} \mathcal{O}_{\tilde{C}} \otimes\left(\mathcal{O}_{x} / m_{x}\right)$ is a complex vector space $V$ of dimension 2, and we have a canonical surjective morphism $\psi: \pi_{*} \mathcal{O}_{\tilde{C}} \rightarrow V$. For $a, b$ not both zero, we construct a quotient

$$
\phi_{a, b}: \pi_{*} \mathcal{O}_{\tilde{C}} \rightarrow \mathbb{C}_{p} \rightarrow 0
$$

as follows.

We assume that the local equation of the curve $C$ around $p$ is given by $x y=0$ with local coordinates $x, y$. Let $\mathcal{O}_{p}$ denote the local ring at $p$ with maximal ideal $m_{p}$, and $\hat{\mathcal{O}}_{p}$ the completion of $\mathcal{O}_{p}$; then $\hat{\mathcal{O}}_{p}=k[[x, y]] /(x y)$, and $\hat{m}_{p}=$ $(x, y) k[[x, y]] /(x y)$.

Since the stalk of $\pi_{*} \mathcal{O}_{\tilde{C}}$ at $p$ is isomorphic to $m_{p}$ as an $\mathcal{O}_{p}$-module, we fix such an isomorphism once and for all. Now we denote the images of $x, y$ under $\psi$ by $e_{1}, e_{2}$ respectively. Every $v \in V$ can be written uniquely as $v=v_{1} e_{1}+v_{2} e_{2}$; hence for $a, b$ not both zero, we define $f_{a, b}: V \rightarrow \mathbb{C}$ to be $f(v)=a v_{1}+b v_{2}$, and it is surjective. Finally we set $\phi_{a, b}=f_{a, b} \psi$.

Proposition 2.1. Using the above notation, we denote the kernel of $\phi_{a, b}$ by $\mathcal{E}_{a, b}$. Then $\mathcal{E}_{a, b}$ is invertible if and only if $a b \neq 0$.

Proof. First we show that if $a b=0$, then $\mathcal{E}_{a, b}$ is not invertible. We can suppose $a=0$. Then $b \neq 0$ and from the definition of $f_{a, b}$, we get that $\operatorname{ker}\left(f_{a, b}\right)$ is the subspace generated by $e_{1}$. Now we denote the completion of the stalk $\left(\mathcal{E}_{a, b}\right)_{p}$ by $\left(\hat{\mathcal{E}}_{a, b}\right)_{p}$; then we have $x \in\left(\hat{\mathcal{E}}_{a, b}\right)_{p}$. But $y \notin\left(\hat{\mathcal{E}}_{a, b}\right)_{p}$, and $y^{2} \in\left(\hat{\mathcal{E}}_{a, b}\right)_{p}$. Thus $\left(\hat{\mathcal{E}}_{a, b}\right)_{p}$ is not a free $\hat{\mathcal{O}}_{p}$-module, i.e., $\mathcal{E}_{a, b}$ is not invertible.

Conversely, we follow the similar argument. From $a b \neq 0$, we know that $V$ is generated by $b e_{1}-a e_{2}$. Hence $\left(\hat{\mathcal{E}}_{a, b}\right)_{p}$ is isomorphic to $\hat{\mathcal{O}}_{p}$ as a module, i.e., $\mathcal{E}_{a, b}$ is invertible.

It is obvious that for invertible sheaves $\mathcal{E}_{a_{1}, b_{1}}$ and $\mathcal{E}_{a_{2}, b_{2}}$ we have $\mathcal{E}_{a_{1}, b_{1}} \cong \mathcal{E}_{a_{2}, b_{2}}$ if and only if $a_{1}: b_{1}=a_{2}: b_{2}$, and every invertible sheaf can be obtained from this process. Hence the Jacobian of $C$ is $\mathbb{C}^{*}$.

\section{General FaCts}

In this section, we give some general facts related to the construction of rank 2 stable sheaves on a rational nodal curve. 
Let $C$ be a rational nodal curve and let $p$ be one of the nodes on it. Let $\pi: \tilde{C} \rightarrow C$ be the partial normalization of $C$ at $p$. The inverse image of $p$ is denoted by $q_{1}, q_{2}$. For the node $p \in C$, let $\mathcal{O}_{p}$ be its local ring with $m_{p}$ the maximal ideal.

Lemma 1.5 leads to the following definition.

Definition 3.1. A rank 2 torsion free sheaf $\mathcal{E}$ on $C$ is said to be of type $a$ at $p$, for $0 \leq a \leq 2$, if we have

$$
\mathcal{E}_{p} \cong a \mathcal{O}_{p} \oplus(2-a) m_{p} .
$$

When $p$ is the only node on $C$, we simply say a sheaf is of type $a$, and type 2 sheaves are just locally free sheaves.

In the sequel, we focus on sheaves of type 1 at $p$.

Lemma 3.2. Every rank 2 sheaf $\mathcal{E}$ of type 1 at $p$ canonically fits into the following exact sequence:

$$
0 \rightarrow \mathcal{E} \rightarrow \pi_{*} \tilde{\mathcal{E}} \rightarrow \mathbb{C}_{p} \rightarrow 0
$$

where $\tilde{\mathcal{E}}$ is a torsion free sheaf on $\tilde{C}$ and it is free at $q_{1}, q_{2}$; such an $\tilde{\mathcal{E}}$ is unique up to isomorphism. Furthermore, every automorphism of $\mathcal{E}$ is induced by an automorphism of $\pi_{*} \tilde{\mathcal{E}}$.

Proof. For any torsion free sheaf $\mathcal{E}$ on $C$, we define a skyscraper sheaf $\mathcal{T}$ supported at $p$ by the exact sequence

$$
0 \rightarrow \mathcal{E} \rightarrow \pi_{*} \pi^{*} \mathcal{E} \rightarrow \mathcal{T} \rightarrow 0 .
$$

Obviously, $\chi(\mathcal{T})=2$.

In fact, $\pi^{*} \mathcal{E}$ is not a torsion free $\mathcal{O}_{\tilde{C}}$-module; so we let $\tilde{\mathcal{E}}=\pi^{*} \mathcal{E} / \operatorname{Tor}\left(\pi^{*} \mathcal{E}\right)$. Since $\mathcal{E}$ is torsion free, we have

$$
0 \rightarrow \mathcal{E} \rightarrow \pi_{*} \tilde{\mathcal{E}} \rightarrow \mathcal{Q} \rightarrow 0
$$

where $\mathcal{Q}$ is a skyscraper sheaf supported at $p$ and $\chi(\mathcal{Q})<\chi(\mathcal{T})$. From Lemma 1.6, $\pi_{*} \tilde{\mathcal{E}}$ is of type 0 at $p$, thus is not isomorphic to $\mathcal{E}$. This means $\mathcal{Q}$ is not a zero sheaf; hence $\chi(\mathcal{Q})=1$, and $\mathcal{Q}=\mathbb{C}_{p}$.

Now, given an exact sequence

$$
0 \rightarrow \mathcal{E} \rightarrow \pi_{*} \mathcal{F} \rightarrow \mathbb{C}_{p} \rightarrow 0
$$

by applying $\pi^{*}$ and $\pi_{*}$, and noting that $\pi_{*} \pi^{*} \pi_{*} \mathcal{F} \cong \pi_{*} \mathcal{F}$, we obtain an injective homomorphism

$$
\pi_{*} \tilde{\mathcal{E}} \rightarrow \pi_{*} \mathcal{F}
$$

since they have the same Euler characteristics, it is an isomorphism.

Similarly, given an automorphism $\psi: \mathcal{E} \rightarrow \mathcal{E}$, apply $\pi^{*}$ and $\pi_{*}$ on both sides, and module the torsion parts. Then we get an automorphism of $\pi_{*} \tilde{\mathcal{E}}$.

Unfortunately, given such a quotient

$$
\pi_{*} \tilde{\mathcal{E}} \stackrel{\rho}{\rightarrow} \mathbb{C}_{p} \rightarrow 0,
$$

the kernel $\mathcal{K}=\operatorname{ker}(\rho)$ is not always of type 1 at $p$. In order to find the condition on $\rho$ such that $\mathcal{K}$ is of type 1 at $p$, we must analyze the quotient in detail.

Following the argument of the Jacobian of $C$, we use local coordinates to give an explicit form of the quotient. Recall that we can assume that the local equation of the curve $C$ around $p$ is given by $x y=0$ with local coordinates $x, y$. Let $\hat{\mathcal{O}}_{p}$ 
be the completion of $\mathcal{O}_{p}$. $\hat{\mathcal{O}}_{p}$ is a complete local ring with maximal ideal $\hat{m}_{p}$, and $\hat{\mathcal{O}}_{p} \cong k[[x, y]] /(x y), \hat{m}_{p} \cong(x, y) k[[x, y]] /(x y)$.

As an $\mathcal{O}_{p}$-module the stalk of $\pi_{*} \tilde{\mathcal{E}}$ at $p$ is isomorphic to $m_{p} \oplus m_{p}$. We fix such an isomorphism and identify them in the sequel. Now we denote by $m_{1}, m_{2}$ the two summands of the completion of $\left(\pi_{*} \tilde{\mathcal{E}}\right)_{p}$, with $m_{1}=\left(x_{1}, y_{1}\right) k\left[\left[x_{1}, y_{1}\right]\right] /\left(x_{1} y_{1}\right)$, $m_{2}=\left(x_{2}, y_{2}\right) k\left[\left[x_{2}, y_{2}\right]\right] /\left(x_{2} y_{2}\right)$. We have a canonical homomorphism $\phi: m_{1} \oplus$ $m_{2} \rightarrow m_{1} / m_{1}^{2} \oplus m_{2} / m_{2}^{2}$, where $m_{i} / m_{i}^{2}$ is a $\mathbb{C}$-vector space $V_{i}$ of dimension 2. Let $V=V_{1} \oplus V_{2}$; then every nonzero linear form $u$ on $V$ gives rise to a quotient $\rho=u \phi$, and conversely, every quotient can be induced from a linear form $u$ on $V$. In the following, we shall identify quotients $\rho: \pi_{*} \tilde{\mathcal{E}} \rightarrow \mathbb{C}_{p} \rightarrow 0$ with nonzero forms on $V$.

Since $V=V_{1} \oplus V_{2}$, with $V_{i}=m_{i} / m_{i}^{2}$, and $m_{i}$ is generated by $x_{i}, y_{i}$, it follows that the image of $x_{1}, y_{1}, x_{2}, y_{2}$ under the canonical map $\phi: m_{1} \oplus m_{2} \rightarrow V_{1} \oplus V_{2}$, denoted by $e_{1}, f_{1}, e_{2}, f_{2}$, forms a basis of $V$. Now let $W=V^{*}$ be the space of linear forms on $V$. The dual basis in $W$ is denoted by $e_{1}^{*}, f_{1}^{*}, e_{2}^{*}, f_{2}^{*}$. In the sequel, we fix the basis of $V$ and $W$, and $v=\left(v_{1}, w_{1}, v_{2}, w_{2}\right)^{T} \in V$ means that $v=$ $\left(e_{1}, f_{1}, e_{2}, f_{2}\right)\left(v_{1}, w_{1}, v_{2}, w_{2}\right)^{T}$.

Now let $u \in W \backslash 0$ be a nonzero linear form on $V$ given by

$$
\begin{aligned}
& u\left(e_{i}\right)=\alpha_{i}, \\
& u\left(f_{i}\right)=\beta_{i} ;
\end{aligned}
$$

then $u=\left(e_{1}^{*}, f_{1}^{*}, e_{2}^{*}, f_{2}^{*}\right)\left(\alpha_{1}, \beta_{1}, \alpha_{2}, \beta_{2}\right)^{T}$, or simply $u=\left(\alpha_{1}, \beta_{1}, \alpha_{2}, \beta_{2}\right)^{T}$. We have

Lemma 3.3. Given a quotient

$$
\pi_{*} \tilde{\mathcal{E}} \stackrel{\rho}{\rightarrow} \mathbb{C}_{p} \rightarrow 0
$$

such that $\rho=u \phi$ and $u=\left(\alpha_{1}, \beta_{1}, \alpha_{2}, \beta_{2}\right)^{T}$, the kernel $\mathcal{K}=\operatorname{ker}(\rho)$ is of type 0 at $p$ if and only if $\left(\alpha_{1}, \alpha_{2}\right)=0$ or $\left(\beta_{1}, \beta_{2}\right)=0$; otherwise, $\mathcal{K}$ is of type 1 at $p$.

Proof. Suppose we are given a quotient

$$
\pi_{*} \tilde{\mathcal{E}} \stackrel{\rho}{\rightarrow} \mathbb{C}_{p} \rightarrow 0 .
$$

The type of the sheaf $\mathcal{K}=\operatorname{ker}(\rho)$ at $p$ is a completely local property, and it does not depend on the isomorphism $\left(\pi_{*} \tilde{\mathcal{E}}\right)_{p} \cong m_{p} \oplus m_{p}$. In fact, we can always find an isomorphism under which a quotient has the form $u=\left(\alpha_{1}, \beta_{1}, \alpha_{2}, \beta_{2}\right)^{T}$ such that there are two zero elements.

Suppose that under the isomorphism $\psi:\left(\pi_{*} \tilde{\mathcal{E}}\right)_{p} \cong m_{p} \oplus m_{p}$ we have $u=$ $\left(0, \beta_{1}, 0, \beta_{2}\right)^{T}$. Then $\mathcal{K}_{p}$ is the submodule of $m_{p} \oplus m_{p}$ consisting of elements $\left(x_{1}, y_{1}, x_{2}, y_{2}\right)$ such that $\left(x_{1}, y_{1}, x_{2}, y_{2}\right) u=0$, i.e., $\beta_{1} y_{1}+\beta_{2} y_{2}=0$. In fact, $\mathcal{K}_{p}$ is the direct sum of two submodules $M_{1}, M_{2}$, such that $M_{1}$ is generated by $x_{1}, y_{1}^{2}$, and $M_{2}$ is generated by $x_{2},-\beta_{2} y_{1}+\beta_{1} y_{2}$; obviously $M_{1}$ and $M_{2}$ are both isomorphic to $m_{p}$, and hence $\mathcal{K}$ is of type 0 at $p$.

Actually, this argument can also be applied to other cases.

Suppose we are given a quotient

$$
\pi_{*} \tilde{\mathcal{E}} \stackrel{\rho}{\rightarrow} \mathbb{C}_{p} \rightarrow 0 .
$$

Now we come to study the stability of the kernel $\mathcal{K}$ under the assumption that $\chi(\tilde{\mathcal{E}})$ is even. The following two claims are obvious.

1. $\pi_{*} \tilde{\mathcal{E}}$ stable implies $\mathcal{E}$ stable. 
2. $\pi_{*} \tilde{\mathcal{E}}$ unstable implies $\mathcal{E}$ unstable.

Now suppose $\pi_{*} \tilde{\mathcal{E}}$ is strictly semistable. Then there exists an exact sequence

$$
0 \rightarrow \mathcal{L} \stackrel{s}{\rightarrow} \pi_{*} \tilde{\mathcal{E}} \rightarrow \mathcal{L}^{\prime} \rightarrow 0
$$

such that $\chi(\mathcal{L})=\chi\left(\mathcal{L}^{\prime}\right)=\frac{1}{2} \chi(\tilde{\mathcal{E}})$. Furthermore, the exact sequence is unique if $\pi_{*} \tilde{\mathcal{E}}$ is indecomposable.

Lemma 3.4. If $\pi_{*} \tilde{\mathcal{E}}$ is strictly semistable, then $\mathcal{K}$ is unstable if and only if there exists an injection $s: \mathcal{L} \rightarrow \pi_{*} \tilde{\mathcal{E}}$ such that $\chi(\mathcal{L})=\frac{1}{2} \chi(\tilde{\mathcal{E}})$ and $\rho s=0$.

Proof. If $\rho s=0$, we have an injection $s^{\prime}: \mathcal{L} \rightarrow \mathcal{K}$ such that the composition of $s^{\prime}$ and $\mathcal{K} \rightarrow \pi_{*} \tilde{\mathcal{E}}$ is just $s ;$ since $\chi(\mathcal{L})=\frac{1}{2} \chi(\tilde{\mathcal{E}})>\frac{1}{2} \chi(\mathcal{K}), \mathcal{K}$ is unstable.

Conversely, if $\mathcal{K}$ is unstable, then we have a rank 1 subsheaf $\mathcal{L}$ of $\mathcal{K}$ such that $\chi(\mathcal{L})>\frac{1}{2} \chi(\mathcal{K})$; followed by $\mathcal{K} \rightarrow \pi_{*} \tilde{\mathcal{E}}$, we get an injection $s: \mathcal{L} \rightarrow \pi_{*} \tilde{\mathcal{E}}$. Since $\pi_{*} \tilde{\mathcal{E}}$ is strictly semistable, we get $\chi(\mathcal{L})=\frac{1}{2} \chi(\tilde{\mathcal{E}})$ and $\rho s=0$.

\section{Rank 2 Stable Sheaves on a Rational CURVE With one Node}

Let $C$ be a rational nodal curve with only one node $p$, and $\pi: \tilde{C} \rightarrow C$ the normalization of $C$. Denote by $\mathbf{M}$ the moduli space of rank 2 stable sheaves $\mathcal{E}$ on $C$ such that $\chi(\mathcal{E})=1$. In this section, we show that $e(\mathbf{M})=1$. Recall that for every torsion free sheaf $\mathcal{E}$ on $C$ we have $\mathcal{E}_{p} \cong a \mathcal{O}_{p} \oplus(2-a) m_{p}$, for some $a, 0 \leq a \leq 2$, and $m_{p}$ is the maximal ideal of $\mathcal{O}_{p}$.

The moduli space $\mathbf{M}$ can be stratified into three strata $\mathbf{M}^{a}$, where the stratum $\mathbf{M}^{a}$ is the set of stable sheaves of type $a$. From the stratification, we have $e(\mathbf{M})=$ $\sum e\left(\mathbf{M}^{a}\right)$. Next we shall calculate $e\left(\mathbf{M}^{a}\right)$ one-by-one.

1. $\mathbf{M}^{0}$ is empty.

Every sheaf $\mathcal{E}$ in $\mathbf{M}^{0}$ is a direct image of a locally free sheaf $\mathcal{F}$ on $\tilde{C}$, in symbols

$$
\mathcal{E} \cong \pi_{*} \mathcal{F}
$$

By virtue of Grothendieck's lemma, any locally free sheaf on $\mathbb{P}^{1}$ is a direct sum of invertible sheaves, $\mathcal{F} \cong \mathcal{O}(l) \oplus \mathcal{O}(m)$, and $\mathcal{F}$ is certainly not stable. Hence $\mathcal{E}$ is not stable, and we get that $\mathbf{M}^{0}$ is empty.

2. $e\left(\mathbf{M}^{2}\right)=0$.

Every sheaf $\mathcal{E}$ in $\mathbf{M}^{2}$ is locally free. Since the Jacobian $J C$ is $\mathbb{C}^{*}$, for an arbitrary large prime number $p$, there is an element $\mathcal{L}$ of order $p$ in $J C$, i.e., an invertible sheaf $\mathcal{L}$ of degree 0 , such that $\mathcal{L}^{\otimes p}$ is trivial and $\mathcal{L}^{\otimes n}$ is nontrivial for $0<n<p$. Then $\mathcal{L}$ generates a cyclic subgroup $G$ in $J C$. We define a $G$-action $\sigma: G \times \mathbf{M}^{2} \rightarrow \mathbf{M}^{2}$ by

$$
\sigma(\mathcal{L}, \mathcal{E})=\mathcal{E} \otimes \mathcal{L}
$$

Now we show that when $p>2$, the action is free. Suppose $\mathcal{E} \cong \mathcal{E} \otimes \mathcal{L}^{\prime}$ for some $\mathcal{E} \in \mathbf{M}^{2}$ and $\mathcal{L}^{\prime} \in G$. Then $\operatorname{det}(\mathcal{E}) \cong \operatorname{det}\left(\mathcal{E} \otimes \mathcal{L}^{\prime}\right) \cong \operatorname{det}(\mathcal{E}) \otimes \mathcal{L}^{\prime \otimes 2}$, and $\operatorname{det}(\mathcal{E})$ is invertible; hence $\mathcal{L}^{\prime \otimes 2}$ is trivial. But this is impossible since $p$ is prime and $p>2$; thus the action is free. Now by Lemma 1.7, we obtain $e\left(\mathbf{M}^{2}\right)=0$.

Remark 4.1. In fact, we have the following result. Let $\mathcal{V}$ be a rank 2 locally free stable sheaf on $C, \chi(\mathcal{V})=1$. Then $\mathcal{V}$ fits into the exact sequence

$$
0 \rightarrow \mathcal{O}_{C} \rightarrow \mathcal{V} \rightarrow \mathcal{O}_{C}(p) \rightarrow 0
$$

for some point $p$ on $C$ different from $x$. This also shows that $e\left(\mathbf{M}^{2}\right)=0$. 
3. $\mathbf{M}^{1}$ is one point.

From Lemma 3.2, any sheaf in $\mathbf{M}^{1}$ fits into the following exact sequence:

$$
0 \rightarrow \mathcal{E} \rightarrow \pi_{*} \mathcal{O}_{\tilde{C}} \oplus \pi_{*} \mathcal{O}_{\tilde{C}} \rightarrow \mathbb{C}_{p} \rightarrow 0
$$

Since every sheaf in $\mathbf{M}^{1}$ comes from a quotient, we can reconstruct $\mathbf{M}^{1}$ from the space of such quotients. Let $\rho: \pi_{*} \mathcal{O}_{\tilde{C}} \oplus \pi_{*} \mathcal{O}_{\tilde{C}} \stackrel{\rho}{\rightarrow} \mathbb{C}_{p} \rightarrow 0$ be an arbitrary quotient. Denote by $\mathcal{F}$ the kernel of $\rho$; in general, $\mathcal{F}$ is not stable, and it may happen that $\mathcal{F}$ is not of type 1 .

Recall that we have identified quotients $\rho: \pi_{*} \mathcal{O}_{\tilde{C}} \oplus \pi_{*} \mathcal{O}_{\tilde{C}} \rightarrow \mathbb{C}_{p} \rightarrow 0$ with nonzero forms on $V$.

Now let $W=V^{*}$ be the space of linear forms on $V$, and let $S$ be the set of isomorphic classes of rank 2 sheaves $\mathcal{E}$ on $C$ such that $\chi(\mathcal{E})=1$.

Since for each quotient

$$
\rho: \pi_{*} \mathcal{O}_{\tilde{C}} \oplus \pi_{*} \mathcal{O}_{\tilde{C}} \rightarrow \mathbb{C}_{p} \rightarrow 0
$$

the kernel of $\rho$, denoted by $\mathcal{K}_{\rho}$, is an element in $S$, we obtain a map $\Psi: W \backslash 0 \rightarrow S$.

In fact, the map $\Psi$ is simple.

The group $A u t_{\mathcal{O}_{C}}\left(\pi_{*} \mathcal{O}_{\tilde{C}} \oplus \pi_{*} \mathcal{O}_{\tilde{C}}\right)$ of automorphisms of the $\mathcal{O}_{C}$-module $\pi_{*} \mathcal{O}_{\tilde{C}} \oplus$ $\pi_{*} \mathcal{O}_{\tilde{C}}$ is canonically isomorphic to Aut $_{\mathcal{O}_{\tilde{C}}}\left(\mathcal{O}_{\tilde{C}} \oplus \mathcal{O}_{\tilde{C}}\right) \cong G L(2, \mathbb{C})$. For convenience, we denote it by $G$.

Since $m_{1} \oplus m_{2}$ is the completion of the stalk $\left(\pi_{*} \mathcal{O}_{\tilde{C}} \oplus \pi_{*} \mathcal{O}_{\tilde{C}}\right)_{p}$, the $G$-action on $\pi_{*} \mathcal{O}_{\tilde{C}} \oplus \pi_{*} \mathcal{O}_{\tilde{C}}$ induces a $G$-action on $m_{1} \oplus m_{2}$, and thus a $G$-action $\sigma$ on $V$. The dual action $\sigma^{\vee}$ on $W$ is defined to be

$$
g u(v))=u(g v)
$$

where $g \in G, u \in W$, and $v \in V$.

Lemma 4.2. Let $\rho_{1}, \rho_{2}$ be two elements in $W \backslash 0$. Then $\Psi\left(\rho_{1}\right)$ and $\Psi\left(\rho_{2}\right)$ are isomorphic as $\mathcal{O}_{C}$-modules if $\rho_{1}, \rho_{2}$ lie in the same orbit of the action $\sigma^{\vee}$.

From this lemma, we know that $\Psi$ is $G$-invariant.

Next, we write down the action $\sigma^{\vee}$ in coordinate form. Recall that $V=V_{1} \oplus V_{2}$, with basis $e_{1}, f_{1}, e_{2}, f_{2}$. The dual basis in $W=V^{*}$ is denoted by $e_{1}^{*}, f_{1}^{*}, e_{2}^{*}, f_{2}^{*}$.

$$
\begin{aligned}
\text { Let } g=\left(\begin{array}{ll}
a & b \\
c & d
\end{array}\right) \in G, v=\left(\begin{array}{c}
v_{1} \\
w_{1} \\
v_{2} \\
w_{2}
\end{array}\right) \in V \text {. Then } \\
\sigma(g, v)=g v=\left(\begin{array}{c}
a v_{1}+b v_{2} \\
a w_{1}+b w_{2} \\
c v_{1}+d v_{2} \\
c w_{1}+d w_{2}
\end{array}\right) .
\end{aligned}
$$


Now let $u=\left(\begin{array}{c}\alpha_{1} \\ \beta_{1} \\ \alpha_{2} \\ \beta_{2}\end{array}\right) \in W \backslash 0$ be a nonzero linear form on $V, g=\left(\begin{array}{ll}a & b \\ c & d\end{array}\right) \in G$. Then

$$
\begin{aligned}
g\left(\begin{array}{l}
\alpha_{1} \\
\beta_{1} \\
\alpha_{2} \\
\beta_{2}
\end{array}\right) & =g u\left(\begin{array}{l}
e_{1} \\
f_{1} \\
e_{2} \\
f_{2}
\end{array}\right)=u g\left(\begin{array}{l}
e_{1} \\
f_{1} \\
e_{2} \\
f_{2}
\end{array}\right)=u\left(\begin{array}{l}
a e_{1}+c e_{2} \\
a f_{1}+c f_{2} \\
b e_{1}+d e_{2} \\
b f_{1}+d f_{2}
\end{array}\right) \\
& =\left(\begin{array}{l}
a \alpha_{1}+c \alpha_{2} \\
a \beta_{1}+c \beta_{2} \\
b \alpha_{1}+d \alpha_{2} \\
b \beta_{1}+d \beta_{2}
\end{array}\right)=\left(\begin{array}{llll}
a & 0 & c & 0 \\
0 & a & 0 & c \\
b & 0 & d & 0 \\
0 & b & 0 & d
\end{array}\right)\left(\begin{array}{l}
\alpha_{1} \\
\beta_{1} \\
\alpha_{2} \\
\beta_{2}
\end{array}\right) .
\end{aligned}
$$

Now we characterize the orbit spaces of the action.

Suppose there are exactly 2 components of $\left(\alpha_{1}, \beta_{1}, \alpha_{2}, \beta_{2}\right)^{T}$ that are zero. They fall into 4 kinds of orbits.

I. $\alpha_{i}=0$, i.e., we have $\left(0, \beta_{1}, 0, \beta_{2}\right)^{T}$. They are all $G$-equivalent; hence there is only one orbit in this case.

II. $\beta_{i}=0$, i.e., we have $\left(\alpha_{1}, 0, \alpha_{2}, 0\right)^{T}$. Also there is only one orbit in this case.

III. $\alpha_{1}=\beta_{1}=0$, or $\alpha_{2}=\beta_{2}=0$, i.e., we have $\left(0,0, \alpha_{2}, \beta_{2}\right)^{T}$ or $\left(\alpha_{1}, \beta_{1}, 0,0\right)^{T}$. Actually every form $\left(0,0, \alpha_{2}, \beta_{2}\right)^{T}$ is equivalent to $\left(\alpha_{2}, \beta_{2}, 0,0\right)^{T}$, but different ratios $\alpha_{2}: \beta_{2}$ give rise to different orbits.

IV. $\alpha_{1}=\beta_{2}=0$, or $\alpha_{2}=\beta_{1}=0$, i.e., we have $\left(0, \beta_{1}, \alpha_{2}, 0\right)^{T}$ or $\left(\alpha_{1}, 0,0, \beta_{2}\right)^{T}$. Clearly there is only one orbit in this case.

In fact, there are exactly these 4 kinds of orbits.

Suppose there are 3 components of $\left(\alpha_{1}, \beta_{1}, \alpha_{2}, \beta_{2}\right)^{T}$ that are zero.

1. If both the $\alpha_{i}$ are zero and one $\beta_{i}$ is not zero, they fall into orbit I.

2 . If both the $\beta_{i}$ are zero and one $\alpha_{i}$ is not zero, they fall into orbit II.

Otherwise, there is at most one component that is zero.

1. If $\left(\alpha_{1}, \beta_{1}, \alpha_{2}, \beta_{2}\right)^{T}$ satisfies $\alpha_{1}: \alpha_{2}=\beta_{1}: \beta_{2}$.

Then there exist $a, c$ such that $a \alpha_{1}+c \alpha_{2}=a \beta_{1}+c \beta_{2}=0$. We pick $b, d$ such that the matrix $\left(\begin{array}{ll}a & b \\ c & d\end{array}\right)$ is invertible and let $g=\left(\begin{array}{ll}a & b \\ c & d\end{array}\right)$. Then

$$
g\left(\alpha_{1}, \beta_{1}, \alpha_{2}, \beta_{2}\right)^{T}=\left(0,0, *_{1}, *_{2}\right)^{T}
$$

with $*_{i} \neq 0$. Therefore, the vector $\left(\alpha_{1}, \beta_{1}, \alpha_{2}, \beta_{2}\right)^{T}$ lies in one of the orbits of case III.

2. If $\left(\alpha_{1}, \beta_{1}, \alpha_{2}, \beta_{2}\right)^{T}$ satisfies $\alpha_{1}: \alpha_{2} \neq \beta_{1}: \beta_{2}$.

Then there exist $a, b, c, d$ such that $a \alpha_{1}+c \alpha_{2}=0, b \beta_{1}+d \beta_{2}=0$. Let $g=\left(\begin{array}{ll}a & b \\ c & d\end{array}\right)$. Then

$$
g\left(\alpha_{1}, \beta_{1}, \alpha_{2}, \beta_{2}\right)^{T}=\left(0, *_{1}, *_{2}, 0\right)^{T}
$$

with $*_{i} \neq 0$. It lies in orbit IV.

Lemma 3.3 and Lemma 3.4 lead to the following two results immediately. 
Corollary 4.3. Let $u$ be an element in $W \backslash 0$. Then $\Psi(u)$ is of type 0 if and only if u lies in orbit I or orbit II. $\Psi(u)$ is of type 1 if and only if u lies in orbit III or orbit $I V$.

Corollary 4.4. If $u$ is an element in $W \backslash 0$, then $\Psi(u)$ is stable if and only if $u$ lies in orbit $I V$.

From Lemma 4.2, there is only one sheaf in $\mathbf{M}^{1}$; a fortiori, $e\left(\mathbf{M}^{1}\right)=1$.

Remark 4.5. From the above result and Remark 4.1, by a little computation, we get that the moduli space $\mathbf{M}$ is a curve isomorphic to $C$ itself.

\section{Proof of the Main theorem}

The following lemma is useful.

Lemma 5.1. Let $C$ be a rational nodal curve with $n$ nodes, and let $\pi: \hat{C} \rightarrow C$ be a partial normalization of $C$ at nodes $x_{1}, \cdots, x_{r}$. Denote by $\mathbf{M}(C)$ the moduli space of rank 2 stable sheaves $\mathcal{E}$ on $C$ such that $\chi(\mathcal{E})=1$. Then $\pi_{*}: \mathcal{E} \rightarrow \pi_{*} \mathcal{E}$ is an injective map from $\mathbf{M}(\hat{C})$ to $\mathbf{M}(C)$.

Proof. Let $\mathcal{E}, \mathcal{F}$ be two rank 2 torsion free sheaves on $\hat{C}$, and $u: \pi_{*} \mathcal{E} \rightarrow \pi_{*} \mathcal{F}$ a homomorphism of $\mathcal{O}_{C}$-modules. We claim that it is in fact $\pi_{*} \mathcal{O}_{\hat{C}}$-linear.

Let $U$ be an affine open subset of $C, s \in \Gamma\left(U, \pi_{*} \mathcal{O}_{\hat{C}}\right), m \in \Gamma\left(U, \pi_{*} \mathcal{E}\right)$. Then $s$ is a rational function on $C$ and can be written as $\frac{a}{b}$, with $a, b \in \Gamma\left(U, \mathcal{O}_{C}\right)$ and $b \neq 0$. The element $u(s m)-s u(m)$ in $\Gamma\left(U, \pi_{*} \mathcal{F}\right)$ is annihilated by $b$, and since $\pi_{*} \mathcal{F}$ is torsion free, we get $u(s m)=s u(m)$, i.e., $u$ is $\pi_{*} \mathcal{O}_{\hat{C}^{-}}$linear.

Next we must show that $\mathcal{E}$ is stable if and only if $\pi_{*} \mathcal{E}$ is stable. The "if" part is trivial. For the "only if" part, suppose $\pi_{*} \mathcal{E}$ is not stable; we show that $\mathcal{E}$ is not stable. We begin with the assumption that $r=1$, that is, $\pi: \hat{C} \rightarrow C$ is a partial normalization of $C$ at one node $x$. Since we suppose $\pi_{*} \mathcal{E}$ is not stable, then there exists a subsheaf $\mathcal{L}^{\prime}$ of $\pi_{*} \mathcal{E}$

$$
0 \rightarrow \mathcal{L}^{\prime} \rightarrow \pi_{*} \mathcal{E} \rightarrow \mathcal{L} \rightarrow 0
$$

with $\mathcal{L}$ torsion free and $\chi(\mathcal{L}) \leq \frac{1}{2} \chi\left(\pi_{*} \mathcal{E}\right)$.

We distinguish two cases.

1. The stalks $\mathcal{L}_{x} \cong m_{x}$; then there is a sheaf $\hat{\mathcal{L}}$ on $\hat{C}$ such that $\mathcal{L} \cong \pi_{*} \hat{\mathcal{L}}$. From the above, $\hat{\mathcal{L}}$ is a quotient sheaf of $\mathcal{E}$, and $\chi(\hat{\mathcal{L}})=\chi(\mathcal{L}) \leq \frac{1}{2} \chi\left(\pi_{*} \mathcal{E}\right)=\frac{1}{2} \chi(\mathcal{E})$. Hence $\mathcal{E}$ is not stable.

2. $\mathcal{L}_{x} \cong \mathcal{O}_{x}$; then we have an exact sequence of $\mathcal{O}_{x}$-modules

$$
0 \rightarrow \mathcal{L}_{x}^{\prime} \rightarrow\left(\pi_{*} \mathcal{E}\right)_{x} \rightarrow \mathcal{L}_{x} \rightarrow 0 .
$$

It is split since $\mathcal{L}_{x} \cong \mathcal{O}_{x}$ is free. Hence $\mathcal{O}_{x}$ is a summand of $\left(\pi_{*} \mathcal{E}\right)_{x}$, which is impossible because $\left(\pi_{*} \mathcal{E}\right)_{x}$ is isomorphic to $m_{x} \oplus m_{x}$.

When $r>1$, we get the result by induction.

Now we consider the Main Theorem. Let $C$ be a rational curve with $n$ nodes $x_{1}, \cdots, x_{n}$. In order to get the result, we only need to calculate $e\left(\mathbf{M}\left(a_{1}, \cdots, a_{n}\right)\right)$ for each stratum, and use $e(\mathbf{M})=\sum e\left(\mathbf{M}\left(a_{1}, \cdots, a_{n}\right)\right)$.

From Lemma $5.1, \mathbf{M}(0, \cdots, 0)$ is empty. The other strata $\mathbf{M}\left(a_{1}, \cdots, a_{n}\right)$ of $\mathbf{M}$ fall into the following three cases.

A. There exists at least one index $i$ such that $a_{i}=2$; that is to say, there is at least one node $x$ such that $\mathcal{E}_{x} \cong \mathcal{O}_{x} \oplus \mathcal{O}_{x}$. 
B. There exist at least two indices $i, j$ such that $a_{i}=a_{j}=1$, and $a_{k}<2$ for all indices $k$.

We have $\sum a_{i} \geq 2$ in these cases. In fact, we shall show that the contribution of such a stratum to the Euler number is 0 . The nonzero contributions come from the cases such that $\sum a_{i}=1$, that is,

C. $\mathcal{E}_{x} \cong m_{x} \oplus m_{x}$ for all but one node $y$, and $\mathcal{E}_{y} \cong \mathcal{O}_{y} \oplus m_{y}$.

Next, we discuss the three cases in turn.

Case $A$. We fix such a stratum $\mathbf{M}_{A}$, and let $\mathcal{E}$ be an element in it. Let $x_{1}, \cdots, x_{r}$ be the nodes of $C$ such that $\mathcal{E}_{x_{i}} \cong \mathcal{O}_{x_{i}} \oplus \mathcal{O}_{x_{i}}, r \geq 1$. Let $y_{j}(1 \leq j \leq s)$ and $z_{k}$ $(1 \leq k \leq t)$ be the nodes such that $\mathcal{E}_{y_{j}} \cong \mathcal{O}_{y_{j}} \oplus m_{y_{j}}, \mathcal{E}_{z_{k}} \cong m_{z_{k}} \oplus m_{z_{k}}$ respectively, $r+s+t=n$, and $s=0, t=0$ are not excluded.

We have an exact sequence of $\mathcal{O}_{C}$-modules

$$
0 \rightarrow \pi_{*} \hat{\mathcal{E}} \rightarrow \mathcal{E} \stackrel{g}{\rightarrow} \oplus \mathbb{C}_{y_{j}} \rightarrow 0
$$

where $\hat{\mathcal{E}}$ is a locally free sheaf on $\hat{C}$ and $\pi: \hat{C} \rightarrow C$ is a partial normalization of $C$ at $y_{j}, z_{k}$. Since $r \geq 1$ (i.e., there exists at least one node $x$ such that $\mathcal{E}$ is free at $x), \hat{C}$ is not smooth. Notice that $\hat{\mathcal{E}}$ is unique up to isomorphism.

Using the fact that $\hat{C}$ is a nodal curve, we can pick a cyclic subgroup $G$ of finite order $p$ in $J C$, for an arbitrary sufficiently large prime $p$, such that for each invertible sheaf $\mathcal{L}$ in $G, \pi^{*} \mathcal{L}$ and $\left(\pi^{*} \mathcal{L}\right)^{\otimes 2}$ are nontrivial. We claim that the $G$ action $\sigma$ on this stratum defined by tensorization is free of fixed points; then by Lemma 1.7, the Euler number is zero.

Now we show that the action $\sigma$ is free. If not, we can find $\mathcal{E}$ such that $\mathcal{E} \otimes \mathcal{L} \cong \mathcal{E}$. From the exact sequence

$$
0 \rightarrow \pi_{*} \hat{\mathcal{E}} \rightarrow \mathcal{E} \stackrel{g}{\rightarrow} \oplus \mathbb{C}_{y_{j}} \rightarrow 0
$$

tensoring by $\mathcal{L}$, we get

$$
0 \rightarrow \pi_{*} \hat{\mathcal{E}} \otimes \mathcal{L} \rightarrow \mathcal{E} \otimes \mathcal{L} \rightarrow \oplus \mathbb{C}_{y_{j}} \rightarrow 0 .
$$

From the isomorphism $\mathcal{E} \otimes \mathcal{L} \cong \mathcal{E}$ and the fact that the kernel of $g$ is unique, we obtain $\pi_{*} \hat{\mathcal{E}} \cong \pi_{*} \hat{\mathcal{E}} \otimes \mathcal{L} \cong \pi_{*}\left(\hat{\mathcal{E}} \otimes \pi^{*} \mathcal{L}\right)$, the second isomorphism being canonical. By Lemma 5.1, $\pi_{*}$ is injective; hence $\hat{\mathcal{E}} \cong \hat{\mathcal{E}} \otimes \pi^{*} \mathcal{L}$. Taking det on both sides, and noticing that $\pi^{*} \mathcal{L}$ and $\left(\pi^{*} \mathcal{L}\right)^{\otimes 2}$ are nontrivial, we get a contradiction.

Case $B$. We fix a stratum $\mathbf{M}_{B}$ in this case, and show that its Euler number is zero.

Let $\mathcal{E}$ be an element in $\mathbf{M}_{B}$; then there are two points, say $x, y$, such that $\mathcal{E}_{x} \cong \mathcal{O}_{x} \oplus m_{x}, \mathcal{E}_{y} \cong \mathcal{O}_{y} \oplus m_{y}$. From Lemma 3.2, every sheaf $\mathcal{E}$ in $\mathbf{M}_{B}$ fits into the exact sequence

$$
0 \rightarrow \mathcal{E} \rightarrow \pi_{*} \tilde{\mathcal{E}} \stackrel{\phi}{\rightarrow} \mathbb{C}_{x} \rightarrow 0
$$

for some sheaf $\tilde{\mathcal{E}}$ on $\tilde{C}$, the partial normalization of $C$ at $x$, and such an $\tilde{\mathcal{E}}$ is unique up to isomorphism.

Now we construct $\mathbf{M}_{B}$ from the space of quotients

$$
\pi_{*} \tilde{\mathcal{E}} \stackrel{\phi}{\rightarrow} \mathbb{C}_{x} \rightarrow 0
$$

Recall that when $\tilde{\mathcal{E}}$ is fixed, we can identify the space of quotients with nonzero elements $u=\left(\alpha_{1}, \beta_{1}, \alpha_{2}, \beta_{2}\right)^{T} \in W$. 
Now we can stratify $\mathbf{M}_{B}$ according to $\tilde{\mathcal{E}}$; that is, every $\tilde{\mathcal{E}}$ gives a stratum $\mathbf{M}_{B}(\tilde{\mathcal{E}})$ whose elements are kernels of the quotients

$$
\pi_{*} \tilde{\mathcal{E}} \stackrel{\phi}{\rightarrow} \mathbb{C}_{x} \rightarrow 0
$$

Denoting by $\mathcal{E}$ the kernel of $\phi$, we analyze $\mathbf{M}_{B}(\tilde{\mathcal{E}})$ for various $\tilde{\mathcal{E}}$.

I. $\pi_{*} \tilde{\mathcal{E}}$ is unstable. Then $\mathcal{E}$ unstable, and hence $\mathbf{M}_{B}(\tilde{\mathcal{E}})$ is empty.

II. $\pi_{*} \tilde{\mathcal{E}}$ is stable. Then $\mathcal{E}$ is always stable. From Lemma $3.3, \mathcal{E}$ is of type 1 if and only if $\left(\alpha_{1}, \alpha_{2}\right) \neq 0$ and $\left(\beta_{1}, \beta_{2}\right) \neq 0$. Since the automorphisms of $\pi_{*} \tilde{\mathcal{E}}$ are multiplications by scalars, and by Lemma $3.2, \mathbf{M}_{B}(\tilde{\mathcal{E}})$ is $\mathbb{P}^{1} \times \mathbb{P}^{1} \times \mathbb{C}^{*}$. Its Euler number is zero.

III. $\pi_{*} \tilde{\mathcal{E}}$ is strictly semistable. Then there exists an exact sequence

$$
0 \rightarrow \mathcal{L} \rightarrow \pi_{*} \tilde{\mathcal{E}} \rightarrow \mathcal{L}^{\prime} \rightarrow 0
$$

such that $\chi(\mathcal{L})=\chi\left(\mathcal{L}^{\prime}\right)=\frac{1}{2} \chi(\tilde{\mathcal{E}})$. Since $\left(\pi_{*} \tilde{\mathcal{E}}\right)_{x} \cong m_{x} \oplus m_{x}$, by tensoring $\mathcal{O}_{x} / m_{x}$ with the above exact sequence, we get that $\mathcal{L}_{x}$ and $\mathcal{L}_{x}^{\prime}$ are both isomorphic to $m_{x}$, i.e., they are direct images of sheaves $\tilde{\mathcal{L}}$ and $\tilde{\mathcal{L}}^{\prime}$ on $\tilde{C}$ respectively.

The case $\pi_{*} \tilde{\mathcal{E}} \cong \mathcal{L} \oplus \mathcal{L}^{\prime}$ and $\mathcal{L} \cong \mathcal{L}^{\prime}$ will never happen, since it contradicts the fact that $\left(\pi_{*} \tilde{\mathcal{E}}\right)_{y} \cong \mathcal{E}_{y} \cong \mathcal{O}_{y} \oplus m_{y}$. Hence $\pi_{*} \tilde{\mathcal{E}}$ is indecomposable, or $\pi_{*} \tilde{\mathcal{E}} \cong \mathcal{L} \oplus \mathcal{L}^{\prime}$ with $\mathcal{L} \neq \mathcal{L}^{\prime}$.

We denote by $\mathbf{M}^{\prime}$ the union of all strata $\mathbf{M}_{B}(\tilde{\mathcal{E}})$ such that $\pi_{*} \tilde{\mathcal{E}}$ is indecomposable. Since there is one sheaf, say $\mathcal{L}$ such that $\mathcal{L}_{y} \cong \mathcal{O}_{y}$, we have a cyclic group $G$ of prime order $p$ in $J C$ such that for every $L \in G, L \otimes \mathcal{L} \neq \mathcal{L}$. From the uniqueness of the exact sequence

$$
0 \rightarrow \mathcal{L} \rightarrow \pi_{*} \tilde{\mathcal{E}} \rightarrow \mathcal{L}^{\prime} \rightarrow 0
$$

we get $L \otimes \pi_{*} \tilde{\mathcal{E}} ¥ \pi_{*} \tilde{\mathcal{E}}$. Hence by Lemma 3.2 the group action of $G$ on $\mathbf{M}^{\prime}$ defined by tensorization is free. By Lemma 1.5 the Euler number $e\left(\mathbf{M}^{\prime}\right)$ is zero.

Finally, $\pi_{*} \tilde{\mathcal{E}} \cong \mathcal{L} \oplus \mathcal{L}^{\prime}$ with $\mathcal{L} \nRightarrow \mathcal{L}^{\prime}$. We have mentioned that $\mathcal{L} \cong \pi_{*} \tilde{\mathcal{L}}$ and $\mathcal{L}^{\prime} \cong \pi_{*} \tilde{\mathcal{L}}^{\prime} ;$ for convenience, we denote $\tilde{\mathcal{L}}$ and $\tilde{\mathcal{L}}^{\prime}$ by $\mathcal{L}$ and $\mathcal{L}^{\prime}$, respectively. With this notation $\tilde{\mathcal{E}} \cong \mathcal{L} \oplus \mathcal{L}^{\prime}$ with $\mathcal{L} \neq \mathcal{L}^{\prime}$. Fix a torsion free sheaf $\mathcal{L}$ on $\tilde{C}$ such that $\chi(\mathcal{L})=1$ and $\mathcal{L}_{y} \cong m_{y}$. We denote by $\mathbf{M}(\mathcal{L})$ the union of all the strata $\mathbf{M}_{B}(\tilde{\mathcal{E}})$ such that $\tilde{\mathcal{E}}$ has a summand $\mathcal{L}$, and define group actions on it.

Since $\tilde{\mathcal{E}}_{y} \cong \mathcal{O}_{y} \oplus m_{y}$ and $\mathcal{L}_{y} \cong m_{y}$, we have $\mathcal{L}_{y}^{\prime} \cong \mathcal{O}_{y}$. From the property of the generalized Jacobian, there is a cyclic group $G$ of order $p$ in $J \tilde{C}$ such that for every $L \in G, L \otimes \mathcal{L} \cong \mathcal{L}$ and $\mathcal{L} \otimes \mathcal{L}^{\prime} ¥ \mathcal{L}^{\prime}$. Then we define a group action of $G$ on $\mathbf{M}(\mathcal{L})$ by tensorization. Obviously $\tilde{\mathcal{E}} \otimes L \neq \tilde{\mathcal{E}}$ for $\tilde{\mathcal{E}} \in \mathbf{M}(\mathcal{L})$ and $L \in G$; by Lemma 3.2, the action is free. But we can choose arbitrarily large $p$ so that the Euler number of $\mathbf{M}(\mathcal{L})$ is zero by Lemma 1.5.

From the stratification given above, we get that $e\left(\mathbf{M}_{B}\right)=0$.

Case $C$. Consider the stratum $\mathbf{M}(1,0, \cdots, 0)$ in this case. Let $\mathcal{E}$ be an element in it. Then $\mathcal{E}_{x_{1}} \cong \mathcal{O}_{x_{1}} \oplus m_{x_{1}}$ and $\mathcal{E}_{x_{i}} \cong m_{x_{i}} \oplus m_{x_{i}}$ for $i>1$. Let $\pi: \hat{C} \rightarrow C$ be the partial normalization of $C$ at all nodes but $x_{1}$. Then $\hat{C}$ is a rational curve with only one node. By Lemma $5.1, \mathbf{M}(1,0, \cdots, 0)$ is just the moduli space of rank 2 stable sheaves $\mathcal{E}$ on $\hat{C}$ such that $\chi(\mathcal{E})=1$. Using the result of $\S 4$, we get $e(\mathbf{M}(1,0, \cdots, 0))=1$.

Now combine these altogether, and noticing that there are $n$ strata of case C, where $n$ is the number of nodes on $C$, we obtain the Main Theorem of this paper. 


\section{ACKNOWLEDGMENT}

I wish to thank Jun Li and Jingen Yang for their guidance and many helpful discussions.

\section{REFERENCES}

[1] A. Beauville. Counting rational curves on K3 surfaces. Duke Math. J. 97 (1999), 99-108. MR 2000c: 14073

[2] A. Grothendieck. Sur la classification des fibrés holomorphes sur la sphère de Riemann. Amer. J. Math. 79 (1957), 121-138. MR 19:315b

[3] R. Hartshorne. Algebraic Geometry. Graduate Texts in Mathematics, No. 52, Springer-Verlag, New York, 1977. MR 57:3116

[4] D. Huybrechts and M. Lehn. The geometry of moduli spaces of sheaves. Vieweg, Braunschweig, 1997. MR 98g:14012

[5] C. S. Seshadri. Fibrés vectoriels sur les courbes algébriques. Astérisque 96 (1982). MR 85b:14023

[6] C. T. Simpson. Moduli of representations of the fundamental group of a smooth projective variety I. Inst. Hautes Études Sci. Publ. Math. 79 (1994), 47-129. MR 96e:14012

Institute of Mathematics, Fudan University, Shanghai 200433, People's Republic of CHINA

E-mail address: wbaosen@etang.com

Current address: Department of Mathematics, Stanford University, Stanford, CA 94305

E-mail address: bwu@math.stanford.edu 